\title{
Efeitos de Intervenção Cognitivo-Comportamental sobre Fatores de Risco Psicológicos em Cardiopatas
}

\author{
Ricardo Gorayeb ${ }^{1}$ \\ Giovana Bovo Facchini \\ Poliana de Lima de Almeida \\ Ana Luisa Magaldi Siguihura \\ Renata Tamie Nakao \\ Andre Schmidt \\ Universidade de São Paulo
}

\begin{abstract}
RESUMO- Diminuir fatores de risco para cardiopatias pode melhorar a qualidade de vida e reduzir a mortalidade e morbidade relacionadas a elas.O presente estudo avaliou a eficácia de uma intervenção cognitivo-comportamental em grupo sobre ansiedade, depressão, estresse e saúde cardiovascular em cardiopatas. Foram avaliados 91 pacientes antes e após participação nos grupos, com entrevistas semiestruturadas, Inventários Beck (Ansiedade e Depressão) e Inventário de Sintomas de Stress para Adultos de Lipp. Coletaram-se medidas fisiológicas para cálculo do escore de Framingham. A participação no grupo resultou em redução dos sintomas de ansiedade, depressão, estresse e melhora do enfrentamento ao estresse. Não houve diferença no escore de Framingham. A intervenção se mostrou eficaz para a redução dos fatores de risco psicológicos nessa amostra.
\end{abstract}

Palavras-chave: doenças cardiovasculares, fatores de risco, intervenção cognitivo-comportamental

\section{Effects in Cognitive-Behavioral Intervention on Psychological Risk Factors of Cardiac Patients}

\begin{abstract}
Reducing risk factors for cardiovascular diseases may increase quality of life and decrease mortality and morbidity secondary to these disorders. The present study evaluated the efficacy of a cognitive-behavioral group intervention on anxiety, depression, stress and cardiovascular health of cardiac patients. Ninety-one patients were assessed before and after the group intervention by means of semi-structured interviews, the Beck Anxiety and Depression Inventories and the Lipp's Stress Symptoms Inventory for Adults. Cardiovascular status was estimated using the Framingham Score. The intervention decreased anxiety, depression, and stress, and improved coping with stress. No significant differences were seen for the Framingham Scores. The intervention was effective in improving psychological risk factors for the investigated sample.
\end{abstract}

Keywords: cardiovascular diseases, risk factors, cognitive-behavioral intervention

As doenças cardiovasculares (DCVs) são a principal causa de morte no mundo. Estima-se que 17,3 milhões de pessoas morreram de DCVs em 2008, representando 30\% de todas as mortes globais. Dessas mortes, 7,3 milhões foram decorrentes de doenças coronárias e 6,2 milhões de acidente vascular cerebral. Países de baixa e média renda são desproporcionalmente afetados, tendo em vista que concentram mais de $80 \%$ das mortes por DCVs. Estima-se que, no ano 2030, aproximadamente 23,6 milhões de pessoas podem morrer de DCVs (World Health Organization, 2012).

As DCVs são altamente prevalentes no Brasil, sendo a causa de mais de 308.000 mortes por ano (DATASUS, 2012a). O conjunto dessas enfermidades constitui a principal causa de gastos em assistência médica, correspondendo a mais de $10 \%$ das internações anuais do sistema público de saúde brasileiro (DATASUS, 2012b). Associa-se sua prevalência ao nível socioeconômico da população, fator que vem sendo considerado inversamente proporcional

1 Endereço para correspondência: Avenida Bandeirantes, 3900, Monte Alegre, Ribeirão Preto, SP, Brasil. CEP: 14.049-900. E-mail: rgorayeb@fmrp.usp.br ao risco de um indivíduo desenvolver uma DCV. Isso possivelmente se explica pelo fato de pessoas com menor renda encontrarem mais obstáculos (dificuldade de acesso a serviços e orientações relacionadas à saúde e custos elevados dos tratamentos) na modificação de comportamentos de risco, como parar de fumar, melhorar a alimentação, realizar atividade física e aderir à medicação (Fiscella \& Tancredi, 2008).

Tem havido muito interesse no estudo do papel dos fatores de risco (FR) para DCVs, pois se entende que é na redução da exposição do indivíduo a eles - especialmente àqueles modificáveis - que se encontra a forma mais eficaz de prevenção e tratamento das cardiopatias (Ford, 2012). Os FR mais citados na literatura são a hipertensão arterial, hipercolesterolemia, diabetes e alguns fatores comportamentais, como alimentação pouco saudável, sedentarismo, excesso de consumo de álcool e tabagismo (Canto et al., 2011; Ford, 2012; Kuklina, Tong, George, \& Bansil, 2012).

Dentre os FR modificáveis, os aspectos emocionais ocupam lugar de grande importância. Depressão, ansiedade, hostilidade, baixo suporte social e estresse crônico vêm 
sendo consistentemente associados ao aumento do risco cardiovascular, segundo uma extensa revisão bibliográfica realizada por Rozanski (2014), a qual analisou metanálises recentes. Essa revisão mostra, também, novos estudos que sugerem associações com outras variáveis psicológicas, tais como pessimismo e falta de sentido na vida, o que indica um crescimento desse campo de pesquisa

Embora as pesquisas sobre a identificação dos FR psicológicos para as cardiopatias estejam bastante avançadas, os achados sobre a melhor estratégia para o manejo desses fatores são ainda inconclusivos (Rozanski, 2014). Intervenções direcionadas à prevenção primária das cardiopatias têm sido relatadas, e envolvem técnicas como orientações nutricionais e de atividade física a indivíduos saudáveis. Entretanto, conclusões sobre a eficácia dessas ações para a redução do risco cardiovascular, bem como da mortalidade de pacientes por eventos cardíacos, ainda não são possíveis (Ladwig et al., 2014).

Maior eficácia é comprovada em estudos que abordam ações psicológicas no âmbito da prevenção secundária, com pacientes já afetados por alguma das DCVs ou com alto risco para desenvolvê-las (Waure et al., 2013). A maioria dos estudos observa impacto positivo dessas ações sobre a qualidade de vida, adoção de padrão comportamental mais saudável e redução no risco de doenças somáticas, inclusive sobre a mortalidade relacionada à cardiopatia (Ladwig et al., 2014; Whalley et al., 2011). Dentre as técnicas psicológicas utilizadas, a Terapia Cognitivo Comportamental (TCC) tem sido largamente relatada e sua eficácia na redução dos FR psicológicos é evidente em diversos estudos internacionais (Barnason, Zimmerman, \& Young, 2011; Berkman et al., 2003; Dekker, Moser, Peden, \& Lenine, 2012; Freedland et al., 2012; Gullikssonet al., 2011; Irvine et al., 2011).

A TCC se mostra uma estratégia adequada para o tratamento e prevenção secundária de pacientes com risco cardíaco, pois, fundamentalmente, oferece modelos comportamentais apropriados para lidar com a doença e o tratamento, auxilia no desenvolvimento de ferramentas para facilitar o manejo das novas demandas e atua sobre as distorções cognitivas, corrigindo aquelas que impedem o adequado controle da doença (Neves \& Molina, 2011). Oferecida em formato grupal, a TCC tem suas vantagens ampliadas, já que possibilita ocorrência de apoio social e se adequa ao serviço público, por prover acesso aos cuidados em um contexto de falta relativa de recursos (Miller et al., 2009).

Embora a TCC venha sendo amplamente investigada com cardiopatas no contexto internacional, com consistência nos resultados obtidos, não foram encontrados estudos com essa intervenção realizados no Brasil. Portanto, é importante realizá-los, pois estudos nacionais podem prover um olhar particularizado sobre pacientes com cultura diferenciada e nível instrucional menor, o que implica em maior risco cardiovascular. Um estudo nacional permitirá também intervir com pacientes usuários de um modelo distinto de assistência à saúde e verificar a eficácia dessas técnicas no contexto sociocultural brasileiro. Assim, o objetivo da presente pesquisa foi avaliar a eficácia de grupos de intervenção com TCC sobre os sintomas de ansiedade, depressão e estresse em pacientes brasileiros com DCV.

\section{Método}

Trata-se de um estudo quase-experimental de corte transversal, com amostra de conveniência constituída de pacientes com DCVs tratados em um hospital público universitário, em uma cidade com cerca de 600 mil habitantes no interior do estado de São Paulo.

\section{Participantes}

Participaram da pesquisa pacientes de ambos os sexos, maiores de 18 anos, com hospitalização prévia no período máximo de dois anos anteriores à entrevista inicial, por diagnóstico de cardiopatias, na Enfermaria Geral de Cardiologia e que, durante a pesquisa, frequentavam os Ambulatórios de Angioplastia, Isquemia, Insuficiência Cardíaca ou Cardiologia Geral da instituição. Por ser o primeiro estudo realizado no Brasil, optou-se por incluir uma amostra geral de pacientes, independentemente da presença de sintomas de ansiedade, depressão e estresse. Esta opção também se deveu ao fato de o presente estudo procurar favorecer e padronizar uma ação assistencial a ser oferecida pelo Serviço de Psicologia aos pacientes cardiopatas da instituição, visando auxiliá-los no manejo de problemas relacionados tanto ao adoecimento quanto a aspectos gerais de suas vidas, como relacionamentos interpessoais, por exemplo. Desse modo, inicialmente não se buscou selecionar os participantes com base em sintomatologias específicas, como as relacionadas a ansiedade, depressão ou estresse, mas sim criar uma intervenção para oferecer o espaço psicoterapêutico para instrumentalização dos pacientes no manejo de seus déficits e/ou excessos comportamentais que pudessem ser FR para suas DCVs. Entende-se que pacientes sem esses sintomas poderiam também se beneficiar da intervenção psicológica, já que os procedimentos abordavam o manejo de problemas cotidianos presentes na vida de todos os indivíduos. Além disso, participar de um grupo de TCC poderia ser benéfico para prevenir o aparecimento de sintomas psicológicos nesses pacientes. Assim, somente foram excluídos os pacientes que apresentassem incapacidade, por alguma doença ou deficiência, de compreender ou responder às questões de forma verbal, clara e consistente.

\section{Instrumentos}

Os dados sociodemográficos e clínicos dos pacientes, bem como o relato oral sobre as estratégias de enfrentamento ao estresse, foram coletados por meio de uma entrevista semiestruturada, elaborada para este estudo e aplicada nas sessões de avaliação pré e pós-intervenção. Para medir presença e intensidade de sintomas de ansiedade, utilizou-se o Inventário Beck de Ansiedade (BAI) (Beck, Epstein, Brown, \& Steer, 1998), cujo coeficiente alfa em amostra brasileira de pacientes cardiopatas é de 0,84 (Cunha, 2001). O Inventário Beck de Depressão (BDI) (Beck, Ward, Mendelson, Mock, \& Erbaugh, 1961) foi utilizado para medir a presença de sintomas de depressão e sua intensidade (coeficiente alfa de 0,77 em amostra brasileira de pacientes cardiopatas) (Cunha, 2001). Analisou-se separadamente a subescala cognitivo- 
afetiva do BDI (BDI 13), pois ela exclui sintomas físicos da depressão que podem ser confundidos com os sintomas da doença cardíaca (Cunha, 2001). O Inventário de Sintomas de Estresse para Adultos de Lipp (ISSL) foi utilizado para avaliar os sintomas físicos e psicológicos de estresse, bem como para classificar a fase de estresse na qual o participante se encontrava: alerta, resistência, quase exaustão ou exaustão (coeficiente alfa de 0,9121) (Lipp, 2000).

Medidas fisiológicas foram colhidas no primeiro (linha de base) e no último dia da intervenção grupal. Foram incluídas medidas de (a) pressão arterial e frequência cardíaca, aferidas duas vezes com o uso de um medidor de pressão arterial automático (Microlife modelo BP A100); (b) peso e altura, aferidos com uma balança mecânica (Filizzola ${ }^{\mathrm{TM}}$ modelo 31 , São Paulo, Brasil) e utilizados para o cálculo do Índice de Massa Corpórea; (c) circunferência abdominal, obtida de acordo com o Manual de Procedimentos Antropométricos do National Health and Nutrition Examination Survey (NHANES), revisado em dezembro de 2000 (National Center for Health Statistics, 2007); e (d) níveis de triglicérides e colesterol, obtidos com o uso de métodos enzimáticos (Wienerlab). O HDL-colesterol foi mensurado por meio do sistema de precipitação das partículas apoB. Todos os métodos utilizados seguem os padrões da Sociedade Brasileira de Análises Clínicas, com variações inferiores a $5 \%$. Algumas medidas fisiológicas foram usadas para o cálculo do escore de Framingham na linha de base e após a intervenção grupal (Anderson, Wilson, Odell, \& Kannel, 1991). O estudo foi desenvolvido em salas especialmente preparadas para o atendimento individual ou de grupos.

\section{Procedimento}

O presente estudo foi aprovado pelo Comitê de Ética em Pesquisas da instituição (Protocolo 13004/2006) e todos os participantes assinaram o Termo de Consentimento Livre e Esclarecido antes do início da intervenção.

O contato com os pacientes se deu pessoalmente na enfermaria e ambulatórios, por telefone ou carta. Caso houvesse aceitação, o termo de consentimento era assinado após o esclarecimento de dúvidas e era realizada uma entrevista inicial com os participantes. Nesse momento eram aplicados os instrumentos de avaliação psicológica. Feito isso, era agendado o início dos grupos, dando seguimento aos mesmos.

Os grupos foram constituídos aleatoriamente de acordo com a disponibilidade de horário dos pacientes, tinham caráter psicoeducativo e psicoterapêutico, com enfoque cognitivo-comportamental. Sua duração foi de 12 sessões semanais, com $2 \mathrm{~h}$ em cada encontro, e se iniciavam com, no mínimo, cinco e, no máximo, 12 pacientes. Foram abordados os temas autoconhecimento, FR e de proteção das doenças cardiovasculares, manejo de estresse, resolução de problemas e modificação de comportamentos relacionados a FR de doenças cardiovasculares. Os grupos foram conduzidos por dois psicólogos, sendo ambos pesquisadores (autores do presente artigo ou bolsistas de Treinamento Técnico em Pesquisa) ou um pesquisador acompanhado por outro psicólogo, com treinamento equivalente. Os temas abordados foram definidos a partir da experiência profissional dos pesquisadores na área e podem ser observados na Tabela 1. Os psicólogos mediavam as discussões, atendendo também demandas dos pacientes que surgiam no decorrer do processo, como outros temas sugeridos pelos integrantes e pertinentes aos objetivos do grupo. Uma descrição detalhada do procedimento terapêutico é apresentada na Tabela 1.

Embora a estrutura da intervenção se baseasse na TCC, os terapeutas também lidaram com as demandas que surgiam durante o processo terapêutico, por vezes incorporando elementos pouco comuns à TCC. Por exemplo, uma sessão com foco na orientação nutricional foi incluída, pois uma

Tabela 1. Descrição de uma intervenção baseada na Terapia Cognitivo-Comportamental

\begin{tabular}{|c|c|}
\hline Sessão & Intervenção \\
\hline $1^{\mathrm{a}}$ & $\begin{array}{l}\text { Apresentação dos terapeutas e dos participantes do grupo, explicação sobre os objetivos da terapia e discussão sobre as } \\
\text { regras para o bom funcionamento do grupo. Estratégias para promover coesão entre os participantes. Foram aferidas as } \\
\text { medidas fisiológicas. }\end{array}$ \\
\hline $2^{\mathrm{a}}$ & Dinâmica de grupo focada em promoção de autoconhecimento. \\
\hline $3^{\mathrm{a}}$ e $4^{\mathrm{a}}$ & $\begin{array}{l}\text { Foco nos fatores de risco e de proteção das DCVs. Foi realizada uma atividade lúdica, em que os participantes } \\
\text { escolheram aleatoriamente figuras representativas de cada fator de risco e discutiram cada uma delas baseada } \\
\text { em conhecimentos prévios adquiridos. Foi solicitado um Registro Semanal de Atividade Física, de acordo com a } \\
\text { recomendação médica, com o objetivo de estimular a atividade física regular. Para os tabagistas que aceitaram a } \\
\text { orientação de parar de fumar, um Registro Semanal de Consumo de Tabaco também foi solicitado, e foram fornecidas } \\
\text { orientações. }\end{array}$ \\
\hline $5^{\mathrm{a}}$ & $\begin{array}{l}\text { O objetivo foi esclarecer dúvidas e discutir sobre alimentação saudável, com a ajuda de uma nutricionista da Divisão de } \\
\text { Cardiologia. }\end{array}$ \\
\hline $6^{\mathrm{a}}$ e $7^{\mathrm{a}}$ & $\begin{array}{l}\text { O grupo discutiu direitos e deveres interpessoais, bem como suas implicações no estresse. Experiências sobre } \\
\text { Direitos Humanos Interpessoais foram apresentadas, seguidas de uma dinâmica de grupo focada em Assertividade. } \\
\text { Comportamentos passivos, assertivos e agressivos foram discutidos no contexto específico de situações- problema. }\end{array}$ \\
\hline $8^{\mathrm{a}}$ a $11^{\mathrm{a}}$ & $\begin{array}{l}\text { Foco na avaliação de contingências, com destaque para a solução de problemas. Foram realizadas dinâmicas de grupo } \\
\text { com conteúdos livres, em que os participantes traziam queixas pessoais para serem discutidas pelo grupo e pelos } \\
\text { terapeutas. }\end{array}$ \\
\hline $12^{\mathrm{a}}$ & Reflexão sobre o processo de aprendizagem e ganhos terapêuticos. Medidas fisiológicas foram novamente aferidas. \\
\hline
\end{tabular}


alimentação inadequada e a obesidade são importantes FR modificáveis para DCVs (Djoussé, Driver, \& Gaziano, 2009; Kannel, 1990), e se observa que certas habilidades cognitivas e comportamentais são necessárias para o desenvolvimento de bons hábitos alimentares (Alvarenga, Scagliusi, \& Philippi, 2008; Ramos \& Stein, 2000). Quanto à sessão em que foram abordados os direitos humanos, o tema foi usado como uma introdução para o Treino de Habilidades Sociais, comumente realizado durante as intervenções comportamentais. Considera-se que esse treino seja fundamental para o bom manejo dos problemas cotidianos que podem predispor ao desenvolvimento de estresse, ansiedade e depressão.

Após a sessão final, eram agendadas as entrevistas individuais de pós-intervenção, quando eram aplicados os mesmos instrumentos da entrevista inicial.

\section{Análise de dados}

Somente foram analisados os resultados dos pacientes que concluíram sua participação na pesquisa, tendo comparecido a, no mínimo, oito sessões do grupo e às entrevistas pré e pós-intervenção.

Os dados são apresentados de forma descritiva, com indicação da frequência e porcentagem de pacientes com sintomas classificados nos diferentes níveis de ansiedade e depressão, bem como nas fases do estresse. Sobre as estratégias de enfrentamento ao estresse, os relatos obtidos foram classificados em dois tipos: (1) "enfrentamento adequado", que incluiu estratégias como procurar distrações ou atividades de lazer, procurar solucionar o problema, falar sobre os sentimentos/ desabafar com alguém de confiança e procurar controlar as emoções, e (2) "enfrentamento inadequado", como evitar falar sobre o problema, brigar, comer em excesso, beber, fumar e não fazer nada. Tinha que haver concordância plena $(100 \%)$ entre dois pesquisadores para que uma classificação fosse adotada.

Utilizou-se análise estatística para verificar significância de diferenças nos resultados dos pacientes antes e após os grupos, sendo aplicado o Teste de Qui quadrado de Pearson $\left(\chi^{2}\right)$ para as variáveis categóricas. Quando a condição necessária para a validade do Qui quadrado foi quebrada (mais de 20\% das frequências esperadas inferiores a cinco), utilizou-se o Teste Exato de Fisher. Para o tratamento de dados numéricos, foi utilizado o teste não paramétrico para amostras relacionadas de Wilcoxon. O critério usado para apontar diferenças estatisticamente significantes foi $p \leq 0,05$.

\section{Resultados}

O processo de constituição da amostra está apresentado na Figura 1. Foram avaliados os prontuários de 1.937 pacientes, dos quais 137 iniciaram sua participação na intervenção, compondo 17 grupos. Desses, 91 concluíram a participação e responderam à entrevista pós-intervenção. A média de frequência nos grupos foi de 10,5 encontros $(D P=2)$.

A média de idade dos pacientes foi de 59,51 anos, variando de 36 a 80 anos, com 6,81 anos de estudo, em média, e 70,32\% $(n=64)$ deles possuíam renda mensal de até

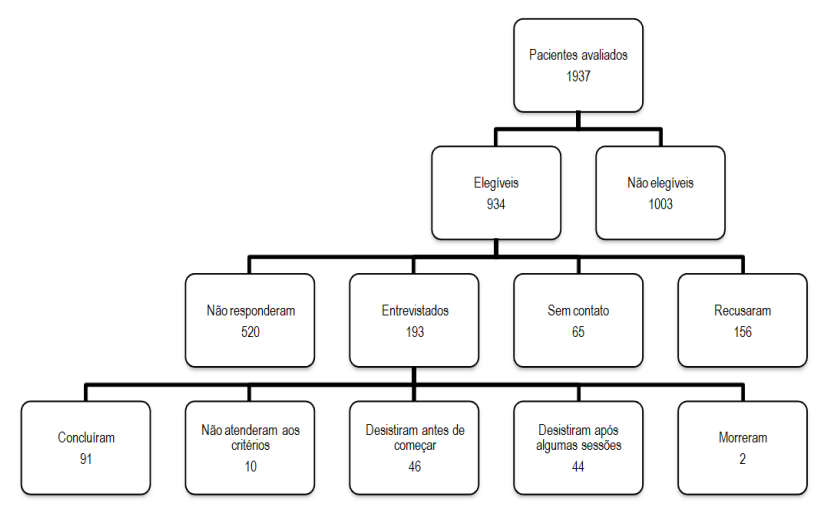

Figura 1. Processo de constituição da amostra com o número de pacientes em cada fase

quatro salários mínimos, com uma média de 2,8 dependentes. Do total, $75,82 \%(n=69)$ eram do sexo masculino. Os participantes casados representaram $60,44 \%$ da amostra $(n$ $=55)$ e $69,3 \%(n=63)$ eram aposentados ou afastados.

Com relação às características clínicas, 78 participantes $(85,71 \%)$ referiram realizar tratamento para hipertensão, $67(73,63 \%)$ realizavam tratamento para dislipidemia e 42 (46,15\%) tinham Diabetes Mellitus. A maioria dos pacientes recebeu o diagnóstico de cardiopatia há menos de dois anos $(50,54 \% ; n=46)$ e, com relação ao comportamento de fumar, $31,87 \%(n=29)$ referiram não ser tabagistas, $52,75 \%(n=48)$ referiram ser ex-tabagistas e $15,38 \%(n=14)$ responderam que fumavam.

A Tabela 2 apresenta a distribuição das frequências e porcentagens de participantes de acordo com seus níveis de ansiedade antes e após a intervenção (Fisher $p<0,001$ ) $\left(\chi^{2}=46,5 ; g l=9\right)$. Houve alteração estatisticamente significante na distribuição dos participantes entre os níveis de ansiedade após a intervenção. Foi possível observar que para 12 participantes $(13,2 \%)$ houve aumento dos níveis de ansiedade, para 54 não houve mudança $(59,3 \%)$ e 25 participantes $(27,5 \%)$ foram classificados em categorias de menor intensidade, indicando redução dos níveis de ansiedade após a participação no grupo.

Além dos dados apresentados na Tabela 2, foi observado que essas diferenças se refletiram na redução da pontuação média de ansiedade na amostra, que variou de 12,37 antes da intervenção para 10,08 após a intervenção $(Z=-3,3 ; p$ $=0,001)$.

Observa-se, na Tabela 3, os dados referentes aos sintomas de depressão, obtidos antes e após a intervenção psicológica. $\mathrm{Na}$ mesma tabela estão apresentados os dados referentes à versão BDI 13, a qual avalia os itens que constituem a subescala cognitivo-afetiva do instrumento, relacionando medidas pré e pós-intervenção com ausência e presença de sintomas cognitivo-afetivos de depressão. Com relação aos níveis de depressão, houve diferença estatisticamente significante na distribuição dos participantes após a participação no grupo. Observou-se aumento no nível de depressão de 17 participantes (18,7\%), enquanto $50(54,9 \%)$ se mantiveram na mesma categoria da avaliação pré-intervenção. Para 24 participantes $(26,4 \%)$, no entanto, o nível de depressão após 
Tabela 2. Frequência e porcentagem de pacientes cardiopatas com sintomas de ansiedade classificados em quatro níveis antes e após a intervenção cognitivo-comportamenal

\begin{tabular}{|c|c|c|c|c|c|c|}
\hline & & & \multicolumn{4}{|c|}{ Pós-intervenção } \\
\hline & & & Mínimo & Leve & Moderado & Severo \\
\hline \multirow{8}{*}{ 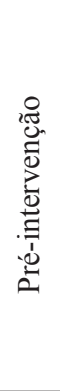 } & \multirow{2}{*}{ Mínimo } & $\mathrm{f}$ & 44 & 5 & 0 & 1 \\
\hline & & $\%$ & 48,4 & 5,5 & 0 & 1,1 \\
\hline & \multirow{2}{*}{ Leve } & $\mathrm{f}$ & 11 & 6 & 3 & 0 \\
\hline & & $\%$ & 12,1 & 6,6 & 3,3 & 0 \\
\hline & \multirow{2}{*}{ Moderado } & $\mathrm{f}$ & 3 & 6 & 1 & 3 \\
\hline & & $\%$ & 3,3 & 6,6 & 1,1 & 3,3 \\
\hline & \multirow{2}{*}{ Severo } & $\mathrm{f}$ & 0 & 3 & 2 & 3 \\
\hline & & $\%$ & 0 & 3,3 & 2,2 & 3,3 \\
\hline
\end{tabular}

a intervenção foi menor do que o inicial (Fisher $p<0,001$ ). Também foi observada diferença estatisticamente significante na comparação das porcentagens na escala reduzida BDI 13 antes a após a participação no grupo, sendo que cinco participantes $(5,5 \%)$ que não apresentavam sintomas afetivocognitivos antes da intervenção passaram a apresentar na avaliação pós-intervenção e, ao contrário, 16 participantes $(17,6 \%)$ passaram da categoria "presença" para a categoria "ausência" de sintomas afeitvo-cognitivos de depressão após o grupo $\left(\chi^{2}=15,6 ; g l=1 ; p<0,001\right)$.

Além da distribuição dos participantes de acordo com os níveis de sintomas de depressão, apresentada na Tabela 3, a média do escore geral de sintomas de depressão, calculada antes e após a intervenção psicológica, também variou de 13,45 para $11,53(\mathrm{Z}=-2,2 ; \mathrm{p}=0,025)$.

Na Tabela 4 estão descritos os resultados obtidos a partir do ISSL, descrevendo as fases de estresse nas quais os participantes se encontravam. A comparação entre a classificação dos participantes nas diversas fases de estresse, efetuada antes e depois da intervenção, indica que houve variações estatisticamente significativas: 58 participantes $(63,7 \%)$ se mantiveram na mesma fase de estresse, nove $(9,9 \%)$ foram classificados em fases superiores àquelas da avaliação pré-intervenção e 24 participantes $(26,4 \%)$ demonstraram redução nos níveis de estresse após a participação no grupo. Destes, destacam-se 12 que foram enquadrados na categoria "resistência" e dois na categoria "exaustão" e que, depois da intervenção, foram classificados como "sem estresse" (Fisher $p<0,001$ ).

Sobre os dados obtidos com o ISSL, também foi analisada a predominância dos tipos de sintomas (físicos ou psicológicos) e observou-se diferença estatisticamente significante na comparação das avaliações antes e após a intervenção. Dois participantes $(2,2 \%)$ que apresentavam predominância de sintomas físicos passaram a ter predominância de sintomas psicológicos e nove participantes $(9,9 \%)$ com predominância de sintomas psicológicos passaram a ter predominância de sintomas físicos (Fisher $p<0,001$ ).

Tabela 3. Frequência e porcentagem de pacientes cardiopatas com sintomas gerais e cognitivo-afetivos de depressão antes e após intervenção cognitivo-comportamental

\begin{tabular}{|c|c|c|c|c|c|c|}
\hline \multicolumn{7}{|c|}{ Sintomas Gerais } \\
\hline & & & \multicolumn{4}{|c|}{ Pós-intervenção } \\
\hline & & & Mínimo & Leve & Moderado & Severo \\
\hline \multirow{8}{*}{ 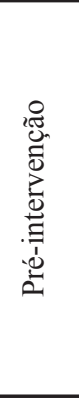 } & \multirow{2}{*}{ Mínimo } & $\mathrm{f}$ & 33 & 7 & 2 & 0 \\
\hline & & $\%$ & 36,3 & 7,7 & 2,2 & 0 \\
\hline & \multirow{2}{*}{ Leve } & $\mathrm{f}$ & 12 & 12 & 6 & 0 \\
\hline & & $\%$ & 13,2 & 13,2 & 6,6 & 0 \\
\hline & \multirow{2}{*}{ Moderado } & $\mathrm{f}$ & 5 & 6 & 4 & 2 \\
\hline & & $\%$ & 5,5 & 6,6 & 4,4 & 2,2 \\
\hline & \multirow{2}{*}{ Severo } & $\mathrm{f}$ & 0 & 1 & 0 & 1 \\
\hline & & & 0 & 1,1 & 0 & 1,1 \\
\hline \multicolumn{7}{|c|}{ Sintomas afetivo-cognitivo } \\
\hline \multirow{5}{*}{ 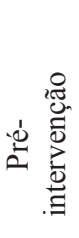 } & & & & & & \\
\hline & \multirow{2}{*}{ Ausência } & $\mathrm{f}$ & \multicolumn{2}{|c|}{58} & \multicolumn{2}{|c|}{5} \\
\hline & & $\%$ & \multicolumn{2}{|c|}{63,7} & \multicolumn{2}{|c|}{5,5} \\
\hline & \multirow{2}{*}{ Presença } & $\mathrm{f}$ & \multicolumn{2}{|c|}{16} & \multicolumn{2}{|c|}{12} \\
\hline & & & \multicolumn{2}{|c|}{17,6} & \multicolumn{2}{|c|}{13,2} \\
\hline
\end{tabular}


A análise realizada em relação aos dois tipos de enfrentamento ao estresse, adequado e inadequado, antes e após a intervenção psicológica indicou redução significante na frequência de relatos de estratégias inadequadas, de 89 na avaliação pré-intervenção para 51 relatos na avaliação pós-intervenção $(\chi 2=10,31 ; g l=1 ; p=0,001)$.

Em relação às medidas fisiológicas, não foram observadas diferenças entre as avaliações pré e pós-intervenção. $\mathrm{O}$ resultados apontam para a relevância de se identificar e buscar tratamentos eficazes para os transtornos psicológicos na população de pacientes cardiopatas, tendo em vista seu impacto sobre o risco de desenvolvimento e agravamento da doença cardíaca.

Em relação à efetividade da intervenção proposta, os resultados encontrados demonstram que as médias dos escores de ansiedade e depressão na amostra foram reduzidas

Tabela 4. Frequência e porcentagem de pacientes cardiopatas com sintomas de estresse, classificados em cinco fases, antes e após intervenção cognitivo-comportamental

\begin{tabular}{|c|c|c|c|c|c|c|c|}
\hline & & & \multicolumn{5}{|c|}{ Pós-intervenção } \\
\hline & & & Sem estresse & Alerta & Resistência & Quase-exaustão & Exaustão \\
\hline \multirow{10}{*}{ 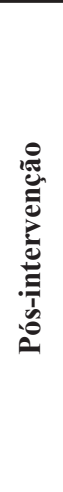 } & Sem estresse & $\mathrm{f}$ & 40 & 1 & 3 & 0 & 0 \\
\hline & & $\%$ & 44,0 & 1,1 & 3,3 & 0 & 0 \\
\hline & Alerta & $\mathrm{f}$ & 0 & 0 & 0 & 0 & 0 \\
\hline & & $\%$ & 0 & 0 & 0 & 0 & 0 \\
\hline & Resistência & $\mathrm{f}$ & 12 & 2 & 15 & 3 & 2 \\
\hline & & $\%$ & 13,2 & 2,2 & 16,5 & 3,3 & 2,2 \\
\hline & Quase- & $\mathrm{f}$ & 1 & 0 & 4 & 3 & 0 \\
\hline & exaustão & $\%$ & 1,1 & 0 & 4,4 & 3,3 & 0 \\
\hline & Exaustão & $\mathrm{f}$ & 2 & 0 & 3 & 0 & 0 \\
\hline & & $\%$ & 2,2 & 0 & 3,3 & 0 & 0 \\
\hline
\end{tabular}

mesmo ocorreu com o escore de Framingham: na avaliação inicial, a média foi de $8,56 \%$ e, na avaliação final, foi de $8,94 \%$, indicando que, nos dois momentos, a média dos participantes se manteve na categoria "baixo risco" (Anderson, Wilson, Odell \& Kannel, 1991).

\section{Discussão}

Os resultados encontrados indicam uma prevalência elevada de transtornos psicológicos na avaliação anterior à intervenção grupal, quando comparada à prevalência em uma amostra não-clínica: no presente estudo, apresentaram sintomas de ansiedade em nível leve, moderado ou severo um total de $45,1 \%$ de participantes, e 53,9\%, apresentaram sintomas de depressão considerando esses três níveis, enquanto que um estudo epidemiológico realizado no município de São Paulo verificou prevalência de 24,95\% de sintomas de ansiedade e/ou depressão em usuários dos serviços de atenção primária à saúde (Maragno, Goldbaum, Gianini, Novaes, \& César, 2006). Todavia, quando comparada com amostras clínicas, a porcentagem de participantes que apresentaram sintomas de ansiedade e depressão nos níveis leve, moderado e severo é semelhante aos níveis de prevalência para esses transtornos obtidos em uma revisão da literatura do período de 1994 a 2009. Nessa revisão, foi observada uma prevalência de até $60 \%$ de depressão e $45 \%$ de ansiedade em populações de pacientes cardiopatas, especialmente aqueles com insuficiência cardíaca (Yohannes, Wilgoss, Baldwin, \& Connolly, 2010). Em conjunto, esses após a realização dos grupos de TCC. Antes da intervenção, as médias (12,37 e 13,45, respectivamente) eram superiores aos escores observados na população brasileira com queixas físicas, obtidas no estudo de padronização e normatização do instrumento (9,94 para ansiedade e 11,41 para depressão) (Cunha, 2001). Os valores médios observados após a intervenção (10,08 e 11,53, respectivamente), por outro lado, foram próximos aos valores padrão. Embora não se possam afirmar efeitos de causalidade por não ter havido controle rigoroso de outras variáveis (eventos de vida adversos tais como problemas financeiros e/ou ocupacionais, dificuldades no relacionamento conjugal e/ou familiar, etc.), a intervenção psicológica pode ter contribuído para esta redução.

Quanto ao estresse, o efeito deletério de seus sintomas vem sendo discutido tanto na literatura nacional (Lipp, Frare, \& Santos, 2007) quanto na internacional (Dimsdale, 2008; Hamer, Molloy,\& Stamatakis, 2008), com ênfase na importância do man ejo dessa variável psicológica para diminuição do sofrimento dos pacientes e incremento em sua qualidade de vida. No presente trabalho, observou-se redução dos sintomas após a intervenção em grupo, com um número estatisticamente significativo de participantes alterando sua forma de enfrentamento e reduzindo a utilização de estratégias ineficazes, como brigar, beber ou fumar. Embora a literatura seja escassa em demonstrar os efeitos de intervenção psicoterápica cognitivo-comportamental sobre sintomas isolados de estresse, alguns trabalhos buscaram corroborar esses resultados (Lipp, 2007; Quintana, 2011).

O pequeno intervalo de tempo (ao redor de 12 semanas) entre a primeira e segunda medida das variáveis fisiológicas, 
como índice de massa corpórea, frequência cardíaca e circunferência abdominal, pode não ter permitido uma observação de alteração das mesmas.Em relação aos dados de pressão arterial e lipidograma, também não se esperavam muitas mudanças, visto estarem próximos da normalidade e também se levando em conta que os pacientes hipertensos têm sua pressão arterial controlada farmacologicamente. Sendo assim, o escore de Framingham também não sofreu alteração já que possui marcadores que demandam longo tempo para modificação, como a dislipidemia e o tabagismo, ou que não são modificáveis, como o diagnóstico de diabetes. Este indicador, portanto, demonstra ter maior utilidade na identificação de pacientes com risco cardiovascular para ações de prevenção das DCVs no contexto clínico, e sua utilização como marcador para avaliar intervenções, especialmente as de menor duração, se mostra limitada (Lotufo, 2008).

Os resultados que apontam redução na proporção de pacientes com sintomas de ansiedade, depressão e estresse em níveis mais graves após a intervenção cognitivocomportamental podem trazer uma contribuição relevante para o tratamento da doença coronariana. Huffman et al. (2011), num estudo prospectivo, observaram que a redução da depressão em pacientes hospitalizados em função de cardiopatia levou a uma melhora clínica significativa e teve impactos promissores no que diz respeito aos efeitos da medicação após a hospitalização. Isso talvez se explique pelo fato de pacientes menos deprimidos terem maiores chances de aderirem ao uso da medicação prescrita e se engajarem em comportamentos mais saudáveis (Bauer et al., 2012).

Como forma de tratamento, o grupo de abordagem cognitivo-comportamental se mostrou eficaz neste estudo. Em revisão bibliográfica, Berben et al. (2011) encontraram que as intervenções mais utilizadas para melhorar a adesão ao tratamento de pacientes cardiopatas são as de caráter educacional, embora estas não sejam mais efetivas do que as intervenções comportamentais que, ainda de acordo com esse estudo, são pouco utilizadas.

No Brasil, a escassez de estudos que tratem de intervenções psicológicas com pacientes cardiopatas revela que talvez pouca atenção esteja sendo dada aos transtornos psicológicos nesse contexto, apesar de suas altas taxas de prevalência nessa população. Estudos brasileiros indicam que as taxas de depressão em pacientes com alguma doença coronária variam de $20 \%$ a 53,3\% (Mattos, Lougon, Tura, \& Pereira, 2005; Oliveira Jr, 2005; Perez, Nicolau, Romano, \& Laranjeira, 2005;), e que os transtornos depressivos estão associados ao aumento da ansiedade e redução na qualidade de vida desses indivíduos (Lemos, Gottschall, Pellanda, \& Müller, 2008).

O presente estudo apresenta algumas limitações, sendo a principal a ausência de um grupo controle. Os resultados, porém, são comparados com uma medida de linha de base, a avaliação antes da intervenção. Outra limitação pode ter sido a elevada taxa de recusa, apresentada no fluxograma, que sugere a possibilidade da amostra conter algum viés, sendo possível que pacientes que aceitaram e concluíram sua participação no estudo possuam algumas características que estejam associadas com as mudanças psicológicas positivas encontradas nos resultados. Ademais, não foram controladas alterações (farmacológicas e não farmacológicas) no tratamento dos pacientes da amostra. Por fim, o fato de se tratar de um hospital público, que atende uma população com baixa renda e pouca escolaridade, pode reduzir o poder de generalização dos dados. Apesar dessas limitações, o trabalho mostrou a importância de se estruturar intervenções psicológicas voltadas aos indivíduos portadores de cardiopatias, demonstrando sua eficácia e favorecendo, assim, o desenvolvimento de estratégias multidisciplinares de cuidado e prevenção, o emprego adequado de recursos públicos para saúde e, principalmente, a melhora na qualidade de vida dos pacientes.

Este trabalho teve como objetivo avaliar a eficácia de intervenção psicológica grupal sobre sintomas de ansiedade, depressão, estresse e medidas fisiológicas em pacientes cardiopatas. A redução na frequência e intensidade de ocorrência dos sintomas psicológicos mostra que é possível obter melhora clinicamente significativa dessas variáveis após um grupo psicoterapêutico de orientação teórica cognitivo-comportamental. Estes dados mostram também a aplicabilidade destes princípios e desta intervenção em um país em desenvolvimento, o que pode refletir favoravelmente no tratamento das cardiopatias.

\section{Referências}

Alvarenga, M. S., Scagliusi, F. B., \&Philippi, S. T. (2008). Changing attitudes, beliefs and feelings towards food in bulimic patients. Archivos Latinoamericanos de Nutrición, 58(3), 274-279.

Anderson, K. M., Wilson, P. W. F., Odell, P. M, \&Kannel, W. B. (1991). An updated coronary risk profile.Circulation, 83, 356-362.

Barnason, S., Zimmerman, L., \& Young, L. (2011).An integrative review of interventions promoting self-care of patients with heart failure. Journal of Clinical Nursing, 21, 448-475.

Bauer, L. K.,Caro, M. A., Beach, S. R., Mastromauro, C. A., Lenihan, E., Januzzi, J. L., \&Huffman, J. C. (2012). Effects of depression and anxiety improvement on adherence to medication and health behaviors in recently hospitalized cardiac patients. American Journal of Cardiology, 109(9), 1266-1271.

Beck, A. T., Epstein, N., Brown, G., \& Steer, R. A. (1998).The Beck Anxiety Inventory. Journal of Consulting and Clinical Psychology, 56, 893-897.

Beck, A. T., Ward, C. H., Mendelson, M., Mock, J., \&Erbaugh, G. (1961). An inventory for measuring depression. Archives of General Psychiatry, 4, 53-63.

Berben, L., Bogert, L., Leventhal, M. E., Fridlund, B., Jaarsma, T., Norekvål, T. M., Smith, K., Strömberg, A., Thompson, D. R., \& ...De Geest, S. (2011). Which interventions are used by health care professionals to enhance medication adherence in cardiovascular patients? A survey of current clinical practice. European Journal of Cardiovascular Nursing, 10(1), 14-21. 
Berkman, L.F., Blumenthal, J., Burg, M., Carney, R.M., Catellier, D., Cowan, M.J., ... Schneiderman, N. (2003). Effects of treating depression and low perceived social support on clinical events after myocardial infarction: The Enhancing Recovery in Coronary Heart Disease Patients (ENRICHD) Randomized Trial. The Journal of the American Medical Association, 289(23), 3106-3116.

Canto, J. G., Kiefe, C.I., Rogers, W. J., Peterson, E. D., Frederick, P. D., French, W. J., Gibson, C. M., PollackJr, C. V., Ornato, J. P., Zalenski, R. J., Penney, J., Tiefenbrunn, A. J.,\& ...Greenland, P. (2011).Number of coronary heart disease risk factors and mortality in patients with first myocardial infarction. The Journal of the American Medical Association, 306(19), 21202127.

Cunha, J. A. (2001). Manual da versão em português das Escalas Beck. São Paulo: Casa do Psicólogo.

DATASUS. (2012a). Taxa de mortalidade especifica por doenças do aparelho circulatório. Banco de dados. Retrievedfromhttp:// www.datasus.gov.br

DATASUS. (2012b). Proporção de internações hospitalares (SUS) por grupo de causas. Banco de dados. Retrievedfromhttp:// www.datasus.gov.br

Dekker, R. L., Moser, D. K., Peden, A. R., \& Lennie, T. A. (2012). Cognitive therapy improves three-month outcomes in hospitalized patients with heart failure. Journal of Cardiac Failure, 18(1), 10-20.

Dimsdale, J. E. (2008). Psychological stress and cardiovascular disease. Journal of the American College of Cardiology, 51(13), 1237-1246.

Djoussé, L., Driver, J. A.,\& Gaziano, J. M. (2009). Relation between modifiable lifestyle factors and lifetime risk of heart failure. JAMA: The Jounal of the American Medical Association, 302(4), 394-400.

Fiscella, K., \&Tancredi, D. (2008). Socioeconomic status and coronary heart disease risk prediction. Journal of the American Medical Association, 300(22), 2666-2668.

Ford, E. S. (2012). Ideal cardiovascular health: Start young, finish strong. Circulation. Retrieved from http://circ.ahajournals.org/

Freedland, K. E., Carney, R. M., Hayano, J., Steinmeyer, B. C., Reese, R. L., \&Roest, A. M. (2012). Effect of obstructive sleep apnea on response to cognitive behavior therapy for depression after an acute myocardial infarction. Journal of Psychosomatic Research, 72, 276-281.

Gulliksson, M., Burell, G., Bessby, B., Lundin, L., Toss, H., \& Svärdsudd, K. (2011). Randomized controlled trial of cognitive behavioral therapy vs standard treatment to prevent recurrent cardiovascular events in patients with coronary heart disease. Archives of Internal Medicine, 171(2),134-140.

Hamer, M., Molloy, G. J., \&Stamatakis, E. (2008). Psychological distress as a risk factor for cardiovascular events: Pathophysiological and behavioral mechanisms. Journal of the American College of Cardiology, 52(25), 2156-62.

Huffman, J. C., Mastromauro, C. A., Sowden, G., Fricchione, G. L., Healy, B. C., \&Januzzi, J. L. (2011). Impact of a depression care management program for hospitalizes cardiac patients. Circulation: Cardiovascular Quality and Outcomes, 4(2), 198-205.
Irvine, J., Firestone, J., Ong, L., Cribbie, R., Dorian, P., Harris, L., Ritvo, P., Katz, J., Newman, D., Cameron, D., Johnson, S., Bilanovic, A., Hill, A., O’Donnell, S., \& ...Sears Jr, S.(2011). A randomized controlled trial of cognitive behavior therapy tailored to psychological adaptation to an implantable cardioverter defibrillator. Psychosomatic Medicine, 73(3), 226-233.

Kannel, W. B. (1990). Contribution of the Framingham Study to Preventive Cardiology. Journal of the American College of Cardiology, 15(1), 206-211.

Kuklina, E. V., Tong, X., George, M. G., \&Bansil, P. (2012). Epidemiology and prevention of stroke: A worldwide perspective. Expert Review of Neurotherapeutics, 12(2), 199-208.

Ladwing, K. H., Lederbogen, F., Albus, C., Angermann, C., Borggrefe, M., Fischer, D., ... Herrmann-Lingen, C. (2014). Position paper on the importance of psychosocial factors in cardiology: Update 2013. German Medical Science, 12, 1-24.

Lemos, C., Gottschall, C. A. M., Pellanda, L. C., \& Müller, M. (2008). Associação entre depressão, ansiedade e qualidade de vida após infarto do miocárdio. Psicologia: Teoria e Pesquisa, 24(4), 471-476.

Lipp, M. E. N. (2000). Inventário de sintomas de stress de Lipp. São Paulo: Casa do Psicólogo.

Lipp, M.E.N. (2007). Controle do estresse e hipertensão arterial sistêmica. Revista Brasileira de Hipertensão, 14( 2), 89-93.

Lipp, M. E. N., Frare, A., \& Santos, F. U. (2007). Efeitos de variáveis psicológicas na reatividade cardiovascular em momentos de stress emocional. Estudos de Psicologia, 24(2), 161-167.

Lotufo, P. A. (2008). O escore de risco de Framingham para doenças cardiovasculares. Revista Medicina (São Paulo), 87(4), 232-237.

Maragno, L., Goldbaum, M., Gianini, R. J., Novaes, H. M. D., \& César, C. L. G. (2006). Prevalência de transtornos mentais comuns em populações atendidas pelo Programa Saúde da Família (QUALIS) no Município de São Paulo, Brasil. Cadernos de Saúde Pública, 22(8), 1639-1648.

Mattos, M. A., Lougon, M., Tura, B. R., \& Pereira, B. B. (2005). Depressão e Síndrome Isquêmica Coronariana Aguda. Revista da Sociedade de Cardiologia do Estado de São Paulo, 18, 288-294.

Miller, W. M., Franklin, B. A., NoriJanosz, K. E., Vial, C., Kaitner, R., \&Mccullough, P. A. (2009). Advantages of group treatment and structures exercise in promoting short-term weight loss and cardiovascular risk reduction in adults with central obesity. Metabolic Syndrome and Related Disorders, 7(5), 441-446.

National Center for Health Statistics. (2007). National Health and Nutrition. Examination Survey. NHANES III, Data, Files, Documentation, and SAS Code. Retrieved from http://www. cdc.gov/nchs/about/major/nhanes/nh3data.htm

Neves, A. S., \& Molina, R. A. (2011). Terapia analítico comportamental e cognitivo-comportamental em grupo: intervenções com portadores de doença crônica. UNICiências, 15(1), 129-140.

Oliveira Jr, W. (2005). Relação médico-paciente em Cardiologia: um olhar psicossomático. Revista de Medicina Psicossomática Sul Mineira, 4, 41-46. 
Perez, G. H., Nicolau, J. C., Romano, B. W., \& Laranjeira, R. (2005). Depressão e síndromes isquêmicas miocárdicas instáveis: diferenças entre homens e mulheres. Arquivos Brasileiros de Cardiologia, 85(5), 319-326.

Quintana, J.F. (2011). A relação entre hipertensão com outros fatores de risco para doenças cardiovasculares e tratamento pela psicoterapia cognitivo-comportamental. Revista SBPH,14(1), 3-17.

Ramos, M., \& Stein, L. (2000). Desenvolvimento do comportamento alimentar infantil. Jornal de Pediatria, 76(3), 229-237.

Rozanski, A. (2014). Behavioral cardiology: Current advances and future directions. Journal of the American College of Cardiology, 64(1), 100-110.

Waure, C., Lauret, G.J., Ricciardi, W., Ferket, B., Teijink, J., Spronk, S., \&Hunink, M. (2013). Lifestyle interventions in patients with coronary heart disease: A systematic review. American Journal of Preventive Medicine, 45(2), 207-216.
Whalley, B., Rees, K., Davies, P., Bennett, P., Ebrahim, S., Liu, Z., ... Taylor, R. S. (2011). Psychological interventions for coronary heart disease. Cochrane Database of Systematic Reviews, 8. Doi:10.1002/14651858.CD002902.pub3

World Health Organization. (2012). Mental health atlas. Recuperado em 16 de maio de 2012, de http://www.who.int/ topics/cardiovascular_diseases/en/.

Yohannes, A. M., Willgoss, T. G., Baldwin, R. C., \& Connolly, M. J. (2010).Depression and anxiety in chronic heart failure and chronic obstructive pulmonary disease: Prevalence, relevance, clinical implications and management principles. International Journal of GeriatricPsychiatry, 25(12), 1209-1221.

Recebido em 28.08.2013

Primeira decisão editorial em 14.01.2015

Versão final em 29.01.2015

Aceito em 23.02.2015 